\title{
Etiology and Chinese Medicine Screening of Severe COVID-19 Based on Multiomics and Serum Pharmacology
}

Yong-Zheng Zhang ( $\triangle$ yongzheng10760@163.com )

Xinjiang Medical University https://orcid.org/0000-0002-8336-0217

\section{ZHANG Yong-Zheng}

Xinjiang Medical University

\section{Meng-Jia}

Xinjiang Medical University

WU Yang

Xinjiang Medical University

\section{Lu-Feng CHENG}

Xinjiang Medical University

\section{Research}

Keywords: Traditional Chinese Medicine, Severe COVID-19, Serum Pharmacology, Network Pharmacology, Omics Analyse

Posted Date: January 12th, 2022

DOI: https://doi.org/10.21203/rs.3.rs-1209452/v1

License: (c) (1) This work is licensed under a Creative Commons Attribution 4.0 International License. Read Full License 


\section{Abstract \\ Background}

At present, scholars believe that severe COVID-19 is related to a variety of basic diseases, and we also observe this point using multi-omics method. The latest sequencing data of severe COVID-19 patients were combined to analyze the pathological mechanism, and pharmacological experimental research on local drugs was conducted, and a compound ingredient was found to have potential medicinal value.

\section{Results}

Here, we observed, for patients with severe COVID-19 disease, the differential miRNA expression is mainly low but having higher expression of mRNA. These differential mRNA expressions are associated with the activation of inflammatory pathways and ultimately with hypoxia and coagulation. Using database analysis, we found that Yi Xin Tong Mai Granule(YXTMG) might regulate COVID-19 through Toll-like receptor signaling pathway by acting on different immune targets. We found a new molecular mechanism for COVID-19 to turn the crisis around, the down-regulated miR-181a-5p mediates the upregulation of PLAU and SERPINE1 molecules to cause cardiovascular adverse events, and YXTMG may prevent it. At the same time, molecular docking indicated that the its various components have antiinflammatory activity. In vitro studies, we confirmed that YXTMG had antioxidant and anti-inflammatory activities.

\section{Conclusions}

The study has supplemented the potential mechanism for the conversion of mild to critical COVID-19 disease and screened the Chinese medicines for improving these factors, providing methodological reference for disease pathology and drug development.

\section{Introduction}

Angina pectoris is the major symptom of coronary atherosclerotic heart disease, while the cardiovascular disease is caused by insufficient coronary artery blood supply, acute and transient myocardial ischemia and hypoxia (R R, et al.,1974). Coronary stenosis is often aggravated by sudden rupture and thrombosis of coronary atherosclerotic plaques in patients, and inflammation and immune response may be the key factors for the rupture of vulnerable plaques ( Nilsson L,et al.,2014;Zhu Yanqi, et al.2012).At present, COVID-19 is still spreading worldwide and has the characteristics of strong appeal, rapid variation, high mortality rate and others. It is clinically found that people with lung or cardiovascular diseases are more likely to develop into severe pneumonia, and severe patients, often dies because of respiratory failure due to immune dysfunction (Metcalf JV, et al.,2020). TongJi hospital, Wuhan, compared the cytokine expression levels of ordinary, severe and critical patients and found that the IL-6 level in the patient's 
blood was positively correlated with the severity of the disease (Panther Chen,et al.,2020).Therefore, for patients with cardiovascular disease in COVID-19, it is crucial and need in the time to develop novel drugs to inhibit the deteriorations and complications caused by the disease. However, anti-inflammatory treatment is an effective method to delay the progression of the disease.

At present, Chinese patent medicine for patients with COVID-19 has been continuously applied in clinical practice, most of which has anti-inflammatory effect. Chinese patent medicine YXTMG is prepare from 8 chinese-medicinal plant species, which are Glehniae radix, Chuanxiong rhizoma, Radix salviae, Hedysarum multijugum maxim, Panax ginseng C. A. Mey, Figwort root, Curcumae radix and Radix glycyrrhizae preparata, it has the effects of replenishing qi, nourishing yin, promotes blood circulation, and removes blood vessels obstruction and platelets aggregation, and is mainly used for management of coronary heart disease like angina pectoris (Jiang Xin,et al.,2012). The constituents such as quercetin and panaxadiol in the prescription given for different treatments have been found to have good antiinflammatory activity (Zhan Jin-shun,et al.,2021;CHEN Yan,et al.,2014;CAI Mei-yun,et al.,2020). While this prescribed medicine can treat severe COVID-19 is still unclear, the study explored the usefulness in context of treatment and potential mechanism of YXTMG, and its active therapeutic entities in the treatment of COVID-19 patients by analyzing gene expression data of severe COVID-19 patients and combining network pharmacology prediction and serum pharmacology with molecular docking.

\section{Materials And Methods}

\section{Database or platform}

\begin{tabular}{ll} 
Database or Platform & Interlinkage \\
\hline GEO & https://www.ncbi.nlm.nih.gov/geo/ \\
\hline TCMSP & https://tcmsp-e.com/ \\
\hline BATMAN & http://bionet.ncpsb.org.cn/ \\
\hline UniProt & https://www.uniprot.org/ \\
\hline Genecards & https://www.genecards.org/ \\
\hline NCBI & https://www.ncbi.nlm.nih.gov/ \\
\hline STRING & https://string db.org/ \\
\hline PubChem & https://pubchem.ncbi.nlm.nih.gov/ \\
\hline PDB & https://www.rcsb.org/
\end{tabular}

\section{Experimental materials}


Fifteen SD rats were provided by the Experimental Animal Center of Xinjiang Medical University. Type $\rrbracket$ alveolar epithelial cells, IL-6/TNF-a/IL-10 ELISA kit, enzyme-linked immunosorbent assay (ELISA) in Shanghai, the batch number is: $\mathrm{m} 1064292-2, \mathrm{~m} 1002859-2, \mathrm{~m} 1002813-2$, respectively, TLR4 primary antibody (bioss), NFKB p65 primary antibody (BioSS), $\beta$-actin primary antibody (Bioss), YiXin TongMai Granule, Ltd., Batch No.2020701, Prednisone acetate tablets, Ltd., Batch No.418001, Quercetin, MCE, Batch No.25265, Fetal bovine serum, Gibco, Trypsin, HyClone, Penicillin-streptomycin, Protein quantitation kit, Biosharp, SDS-PAGE gel making kit, Solarbio, RIPA lysate, Solarbio, $4 \times$ loading buffer, Solarbio. Compound electronic weighing balance, mettler Toledo, Centrifuge, full wavelength microplate reader, $\mathrm{CO}_{2}$ incubator, are Thermo Fisher Scientific company, Fluorescence inverted microscope, leica, germany.

\section{Differential miRNA analysis in critical patients with COVID-19}

The micro-RNA sequencing data of critical COVID-19 patients (GSE176498) were screened in the gene expression profile database, and the blood for miRNA detection results, 10 healthy volunteers and 11 critical COVID-19 patients were selected. The data were read, grouped, corrected, and probe converted to obtain the differential miRNA expression. The analysis results were displayed as heat map, using $\mathrm{R}$ language, and target gene prediction was performed using miRDB and Target-scan, and the intersection of the two parameters was used as the target gene prediction result. Pathway enrichment analysis using miEAA.

\section{Transcriptomics analysis of critical patients}

COVID-19 omics sequencing results were retrieved from the GEO database, and Differential Gene Expression (DGE) analysis was performed on 17 mild cases and 23 critical cases among the sequencing results of critical cases (GSE172114). The volcanic map depicting the overall distribution and the heat map depicting the sample variability were drawn with $\log \mathrm{FC}>3$ as the threshold. In order to further identify the key genes in the disease process of COVID-19, STRING analysis platform was used to analyze the interaction of the differentially expressed genes.

\section{Gene set enrichment analysis and miRNA and mRNA network construction}

In order to identify the pathogenesis of COVID-19, gene set enrichment analysis was performed on the differential genes in the transcriptome using the $\mathrm{R}$ software functional package. Under physiological conditions, there was a negative correlation between the expression of miRNA and mRNA expression level (Ma Shan-shan,et al.,2021). The differential expressions of up-regulated miRNA target genes and downregulated mRNA, and down-regulated miRNA and up-regulated mRNA were intersected and summarized into tables respectively, then the miRNA-mRNA regulatory network was constructed using Cyto3.8.

\section{Principal component and target collection of YXTMG}

Relevant active constituents of YXTMG were collected in a TCMSP database, and oral bioavailability $\geq 30 \%$ and drug-like property $\geq 0.18$ were set as screening criteria to obtain eligible active components 
and targets. The BATMAN database was combined with an online prediction website to collect target information of components not included in the database. Finally, the gene ID was converted using the UniPort database for subsequent network pharmacology studies. The databases of Genecards, DisGeNET, and CTD were used to collect COVID-19 targets using "COVID-19" as the keyword. Inputting targets of COVID-19 and YXTMG into a bioinformatics and evolutionary genomics platform to obtain Venn diagram.

\section{Construction of network and protein interaction network between YXTMG and COVID-19}

The information about the active components, drug targets, diseases, and intersection targets of YXTMG was summarized and imported into Cyto3.8.0 software to draw the network diagram. The components of YXTMG that had effect on the intersection target were classified according to the structure of natural compounds, and the pie chart was drawn using GraphPad. To further clarify the interaction relationship between targets, the intersection targets of YXTMG and COVID-19 were input into the STRING database with the species set as "Homo Sapiens" and the interaction score as high confidence interval (0.900). The results were exported and visualized to obtain the protein network diagram of YXTMG for the treatment of COVID-19.

\section{Gene ontology enrichment and pathway analysis}

The "org.Hs.eg.db" function of R3.6.0 was used for intersection target ID conversion and difference multiple setting, the threshold $P$ was set to be less than $P<0.05$, the "enrichGO" program was used for functional enrichment analysis of biological process, cell composition and molecular function, and the "enrichKEGG" and "pathview" programs were used for KEGG pathway analysis.

\section{Effect of YXTMG on mRNA and miRNA expression in critically ill patients}

The intersection target of YXTMG in the treatment of COVID-19 was selected to intersect with the mRNA and transcription factors of severe patients, and the biological process and pathway analysis of the intersection was performed to identify the effect of YXTMG on the transcription level of patients. The dataset was expanded to clarify the prevalence of differential changes in targets. To intersect the target sites of YXTMG related to miRNA-mediated differential mRNA, and to identify the micro-mechanism in which drugs might shows its therapeutic effect.

\section{Pathological mechanism and combined analysis of critical patients}

In order to identify the intervention pathway of drugs in the treatment of patients with COVID-19, the differential gene enrichment results, miRNA-mediated mRNA enrichment results and YXTMG target of action enrichment information of healthy volunteers from patients with COVID-19 were summarized, and represented by the heat map function of $\mathrm{R}$ software.

\section{Network topology analysis and qualitative and quantitative analysis of quercetin}


To clarify the regulatory effect of YXTMG on immunity, the relationship network between components and innate immunity was constructed and topology analysis was conducted. Qualitative and quantitative analysis of components with good potential was performed by HPLC to clarify the action of active constituents of the drug. Mobile phase consisted of acetonitrile (A) $-0.2 \%$ phosphoric acid (B), with an elution gradient of $(0-12 \mathrm{~min}, 15 \%-24 \% \mathrm{~A}, 12 \rrbracket 16 \mathrm{~min} \varangle 24 \% \varangle 25 \% \mathrm{~A}, 16 \varangle 23 \mathrm{~min} \varangle 25 \% \varangle 26 \% \mathrm{~A}, 23 \varangle 30 \mathrm{~min} \rrbracket$ $26 \% \varangle 48 \% \mathrm{~A}, 30 \varangle 35 \mathrm{~min} \varangle 48 \% \varangle 15 \% \mathrm{~A}$, Flow rate was $1.0 \mathrm{~mL} \cdot \mathrm{min}-1$, The detection wavelength was $254 \mathrm{~nm}$, Column temperature was $30^{\circ} \mathrm{C}$, the injection volume was $10 \mu \mathrm{L}$.

\section{Preparation of medicated serum}

Animal experiments were performed at the Laboratory Animal Center of Xinjiang Medical University, which was certified by AAALAC in 2009. Experiments meet NAP requirements.Fifteen SD rats were randomly divided into the control group, YXTMG group and prednisone group. Normal saline was administered to all Rats intra-gastrically, $20 \mathrm{mg} \cdot \mathrm{kg}^{-1}$ prednisone (Ma Shao dan, et al.,2016) and 894 $\mathrm{mg} \cdot \mathrm{kg}^{-1}$ YXTMG, respectively. The rats were treated intra-gastrically, and administered for three consecutive days two times a day. One hour after the last gavage, rats were narcotized. The abdominal aorta was selected for blood collection. The blood was centrifuged at $3000 \mathrm{rpm} 10 \mathrm{~min}$, sterilized and filtered at $0.22 \mu \mathrm{m}$, inactivated at $56^{\circ} \mathrm{C}$ for $30 \mathrm{~min}$, and frozen at $-80^{\circ} \mathrm{C}$ for standby.

\section{Construction of cell oxidative stress model and drug intervention}

Type $\triangle$ alveolar epithelial cell (AEC囚) in logarithmic phase was plated with 96-well plate at the cell density of $10^{5}$ cells $/ \mathrm{ml}$ and placed in a cell incubator for $4 \mathrm{~h}$ to adhere to the wall. The cells were treated with 10 , $25,50,75,100$ and $125 \mu \mathrm{M} \mathrm{H}_{2} \mathrm{O}_{2}$, respectively. After $4 \mathrm{~h}$, CCK8 solution was added. After incubation for $2 \mathrm{~h}$, the OD value was measured at the wavelength of $450 \mathrm{~nm}$ by a microplate reader. Type II alveolar epithelial oxidative damage cells modeled with $\mathrm{H}_{2} \mathrm{O}_{2}$ were treated with prednisone acetate, YXTMG and quercetincontaining serum, respectively. CCK 8 was added $72 \mathrm{~h}$ after the treated to observe cell activity in each group.

\section{Determination of free radical clearance and IC50 of quercetin}

Oxidative stress response is often associated with the transition to critical illness in patients with COVID19. Therefore, the antioxidant abilities of YXTMG, quercetin and vitamin $C$ were determined using ABTS kit, so as to evaluate the oxygen free radical scavenging ability of the drug. The cell model were achieved by oxidation induced by using hydrogen peroxide with concentrations of $10,30,50,100 \mu \mathrm{M}$ and quercetin with concentration of $200 \mu \mathrm{M}$, and then the absorbance was measured by adding CCK8 reagent after $72 \mathrm{~h}$ to determine the quercetin IC50.

\section{Molecular docking verification}

The 3D structures of TLR4, IRAK4, IKKB, IKBA, and NFKB targets of the COVID-19 signaling pathway were downloaded from the PDB database. The structures of the positive drugs (prednisone) and the active 
components in the top ten of the degree values were downloaded from the PubChem database. The docking tool AutoDockTools was used for semi-flexible docking of all active components and target molecules using the Lamarckian GA algorithm. The lower the binding energy was, the better the binding stability of the components and target molecules was. The results were displayed using the heat map function of R software, and the optimal docking results of the five targets were analyzed and plotted using Pymol software.

\section{Protein imprint verification}

The AEC $₫$ were spread on a six-well plate. After modeling with hydrogen peroxide, the control group, $10 \%$ prednisone, $10 \%$ YXTMG and 50 $\mu \mathrm{M}$ quercetin-containing serum were administered, respectively. The cell suspension was collected for later use after three days. After the cells on the six-well plate were washed, RIPA was used for cell lysis, BCA quantification and protein high-temperature denaturation, and Western Blot was used to conduct electrophoresis and transmembrane operation for each group to detect the relative expression levels of TLR4 and NFKB proteins.

\section{ELISA analysis of inflammatory factor level in cell suspension}

In order to clarify the mechanism of action of drugs, cytokines in each group were tested. The prepared cell suspensions of each group were centrifuged at $1000 \mathrm{rpm}$ for $20 \mathrm{~min}$, and the supernatant was taken for later detection. The IL-6, IL-10, and TNF-a kits were placed at room temperature for equilibration for $60 \mathrm{~min}$. The standard wells, blank wells, and sample wells were set. Then $50 \mu \mathrm{L}$ of the standard and cell suspensions of each group were added, respectively. Next, 100 $\mu \mathrm{L}$ horseradish peroxidase was added. After the sealing of the plate-sealing membrane was performed, the cells were incubated at a constant temperature of $37^{\circ} \mathrm{C}$ for $60 \mathrm{~min}$. The cells were washed five times. After substrates were added into each well, they were incubated in the dark for $15 \mathrm{~min}$. After the stop solution was added, the OD value was measured at a wavelength of $450 \mathrm{~nm}$

\section{Statistical Analysis}

The statistical method in data mining analysis was Bayesian test. The experimental data were all counted in Graphpad 9.0, and the measurement data results were expressed as mean standard error ( $\overline{\mathrm{x}} \pm \mathrm{SE}$ ). One-way analysis of variance was used for comparison among multiple groups, and t-test was used for pairwise comparison within the group. $p<0.05$ indicated that the difference was statistically significant.

\section{Results}

miRNA difference analysis, target gene prediction and heat map construction in critically ill patients with COVID-19

Screening miRNA sequencing data of severe COVID-19 patients in the gene expression profiling database (GSE176498), where's the blood for the miRNA detection, 10 healthy volunteers and 11 severe COVID-19 
patients are selected. Difference analysis revealed 38 up-regulated miRNAs including miR-15a-5p, miR126-5p, miR-29a-3p, miR-29c-3p, and miR-425-5p, and 65 down-regulated miRNAs including miR-92a-3p, miR-26a-5p, miR-150-5p, and miR-181a-5p. Differential miRNAs with LogFC $\geq 3$ were selected, and target gene prediction was performed using two databases and the intersection was taken as a potential target gene, and 3158 up-regulated miRNA target genes and 1912 down-regulated miRNA target genes were obtained. The heat map clustering analysis showed that the miRNA with large differential multiple in severe COVID-19 patients were mainly low-expressed (Fig. 1a).Enrichment analysis of differential miRNA revealed that PI3K-Akt, MAPK, autophagy, cell cycle, chemokines and T cell receptor signaling pathways participated in the development of severe COVID-19 (Fig. 1b).

\section{Transcriptomics analysis, volcanic and thermal mapping of critical patients}

The transcriptome sequencing data of patients with severe pneumonia were obtained after screening. With mild COVID-19 as the control, we found that such differential genes are responsible for the disease development. The results were illustrated as volcanic diagram and it became apparent that the overall distribution of the differential genes was dominated by high mRNA expression. The grouping and clustering analysis of the samples showed that such differential genes as CYP19A1, MMP8, CCNA1, FAP, SLPI, and PPARG were mainly highly expressed in patients with severe COVID-19 (Fig. 2). Transcription factor predictions revealed that 116 transcription molecules including NFKB1, RELA, STAT1, and CEBPB participated in the phenotypic changes of genes in critically ill patients.

\section{Gene Set Enrichment Analysis of DGE and Construction of miRNA and mRNA Network}

To clarify the pathophysiological mechanism of the COVID-19 development in patients, the gene set enrichment analysis of different numbers of up-regulated and down-regulated genes was performed using a "ClusterProfiler" library in R software. The up-regulated genes has a role in the activation of interferon $a / y$, TNF- $a$, complement and inflammatory response signals, and the differential genes jointly inhibited the unfolded protein response and oxidative phosphorylation signals. These results indicated that severe COVID-19 was closely related to endoplasmic reticulum stress, and mitochondrial dysfunction and immune dysfunction (Fig. 3a). Complement system, hypoxia, inflammatory response, IFN-a, TNF-a signals were plotted against the ordered dataset, ranked according to enrichment fraction (Fig. 3b). Mapping of the miRNA and mRNA regulatory network revealed that nine down-regulated miRNAs, including miR-26b-5p and miR-181a-5p, mediated the up-regulation of 31 mRNAs, including PFKFB2, DRAM1 and GRB10. Seven up-regulated miRNA, including miR-126-5p and miR-27a-3p, participated in the down-regulation of 28 mRNA, including RORA, NCALD and CNTNAP2 (Fig. 3c).

\section{Collection of component and targets and disease target of YXTMG}

Ninety-three active compounds of YXTMG, with 2,812 targets, were screened and obtained from the databases of TCMSP and BATMAN. Some compounds are shown in table 1. After being interpreted by the UniProt database and de-duplicated, 83 active components and 757 targets were obtained. Using 
Genecards, NCBI, and TTD databases, and using "COVID-19" as the keyword search target, we obtained 3767 COVID-19 targets.

\section{Venn diagram and protein interaction network draw}

757 targets of YXTMG and 3767 targets of COVID-19 were entered and Venn diagram was drawn on the bioinformatics platform, respectively, and 375 intersecting targets were obtained. Including NFKBIA, RELA, TNF, TLR4, IL6, IL10, EGFR, CXCL10, STAT3, SCARB1, ACE, etc. (Fig.4a). The STRING database was used to analyze the protein-protein interaction relationships of the intersecting targets, and 375 nodes and 1765 interaction relationships were obtained. After setting the parameters, the targets such as SRC, TP53, STAT3, MAPK3, and HSP90AA1 were found to be important target sites for the drug to show the therapeutic effect, after being visualized with Cytoscape software (Fig. 4b).

\section{Drug-target-disease network diagram and pharmacodynamic basic analysis}

The information of active constituents, targets, and intersecting targets was imported into Cytoscape to draw the relationship network of YXTMG, Compounds, Targets and COVID-19(Fig. 5a). Overall, 78 constituents of the eight Chinese medicinal materials act on 375 targets of the COVID-19. As shown in supplement table 1, among the common constituents of YXTMG, the more important in a context of pharmacological effect are quercetin, kaempferol and $\beta$-sitosterol. Constituents classification analysis showed that the those constituents with higher component proportion were successively flavonoids, terpenes, steroids, Alkaloids, and lignans(Fig. 5b), among which, flavonoids were mainly found in Hedysarum Multijugum Maxim, terpenoids were mainly enriched in Curcumae Radix, Figwort Root, and Panax Ginseng C., and the steroidal compounds of the eight Chinese medicines accounted for the same proportion. Alkaloids were mainly found in Panax Ginseng C. A. Mey, (supplement Fig. 1).

\section{Results of GO enrichment analysis and KEGG pathway analysis}

GO and KEGG enrichment analysis was performed using R software, and gene ontology analysis resulted in 3,209 biological processes, 144 cellular pathways and 246 molecular functions. Biological processes were enriched to include responses to lipopolysaccharide, reactive oxygen metabolism, and oxidative stress response, regulation of reactive oxygen metabolism, leukocyte migration, and cell response to drugs, (Fig. 6a). Kyoto gene and genome analysis revealed 191 pathways of information, including PI3KAkt, MAPK, CAMP, TNF, HIF-1 and other environmental information processing as well as tissue systems such as chemokines, Toll-like receptor, C-type lectin receptor and $\mathrm{T}$ cell receptor and their involvement in the incidence of COVID-19 disease, as shown in Fig. $6 \mathrm{~b}$.

\section{Effect of YXTMG on pathological mechanisms of mRNA and miRNA involvement in severe patients}

Through intersection analysis of differential mRNA between drug targets and patient's transcriptome, it was found that the drug might affect MAPK signaling pathway, Toll-like receptor signaling pathway, Ctype lectin receptor signaling pathway, HIF-1 signaling pathway, cell cycle and other biological processes by regulating such pathways like cytokine production regulation, inflammation response regulation, 
epithelial cell proliferation regulation, and DNA binding regulation. The results are shown in supplement fig. 2.In the analysis data set, miR-181a-5p expression was down-regulated. While its target genes PLAU and SERPINE1 were up-regulated(fig.7a).The above phenomena were validated on an expanded dataset based on ARDS symptoms in critically ill patients with COVID-19, and the results were as expected(fig.7b). The intersection of miRNA-induced mRNA expression difference and drug action reveals that stigmasterol and quercetin of Panax ginseng C. A. Mey, Glehniae radix, Hedysarum multijugum maxim act on the down-regulated miR-181a-5p-mediated mRNA up-regulation of PLAU and SERPINE1, and the mRNA expression levels of the two groups, the severe patients are significantly higher than those in mild patients $(\mathrm{p}<0.01)$ (fig.7c). Molecular docking indicated that stigmasterol and quercetin both had good binding with two target molecules (supplement table 2, supplement Fig. 4).

\section{Heat map for combined analysis of pathological mechanism and drug action mechanism in severe COVID-19 patients}

The combined analysis of differential miRNA, mRNA and drug action targets revealed that drugs may affect the regulation of acute inflammatory response, $T$ cell-mediated immunity, humoral immune response and other biological processes in severe COVID-19 patients through such pathways as complement system and coagulation cascade, TNF signaling pathway, NF-kB signaling pathway, T cell receptor signaling pathway, and natural killer cell-mediated cytotoxicity. It is possible that PI3K-Akt signaling pathway can interfere with miRNA-mediated $\alpha-\beta$ T cell differentiation, epithelial cell differentiation regulation (fig.8b), response to oxygen levels, and its role in the hypoxic conditions of biological processes (fig.8a), therefore its efficacy against the severe Covid-19 conditions is inevitable.

\section{Network topology result and qualitative and quantitative analysis of quercetin}

The network topology analysis showed that the constituents such as Quercetin, Kaempferol and Betasitosterol had high central freedom(Table 2), and they could be used as the important material basis of the compound. First, the standard curve(fig.9a) of quercetin was drawn, and the linearity(fig.9c) achieved of quercetin was good within the range of $50-350 \mu \mathrm{g} / \mathrm{mL}$. Qualitative and quantitative analysis of quercetin in YXTMG showed that the compound constituted this component at a high concentration(Table 3 ) of about $1.12 \mathrm{mg} / \mathrm{g}$.

\section{Establishment of cell inflammation model and effect of drugs on oxidative damage cells}

Using a model of inflammatory injury induced by hydrogen peroxide at different concentrations, it was found that the cell inhibition rate was concentration-dependent for hydrogen peroxide, and the inhibition

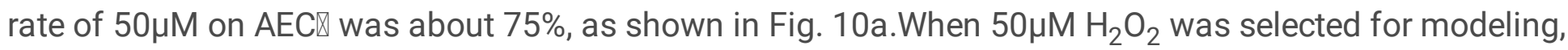
the survival rate of the model group was significantly decreased as compared with that of the control group ( $p<0.01)$. The cell activity of the prednisone group was increased as compared with that of the model group $(p<0.05)$, and the cell activities of quercetin and YXTMG group were significantly increased as compared with that of the model group $(p<0.01)$ (Fig. 10b). 


\section{Evaluation of oxygen free radical scavenge ability and determination of quercetin IC50}

The oxygen free radical scavenging abilities of vitamin C, YXTMG and quercetin were evaluated, and it was found that both the traditional Chinese medicine compound and the active monomer compound had good antioxidant capacity (Fig. 11a). At the concentration of $5 \mathrm{mg} \mathrm{mL}^{-1}$, the antioxidant capacity was ranked as YXTMG, vitamin $C$, and quercetin in descending order of strength. The IC50 curve was illustrated and it was found that the $1 / 2$ of the actual concentration (responsible for activity) of quercetin improved the cells damaged by oxidative stress, which was $7.07 \mu \mathrm{M}$ (fig.11b).

\section{Molecular docking verification results}

The 3D structures of protein and drug molecules were downloaded in the database, and the docking was performed using ADT4.2 tool. The docking results were displayed in the form of heat map. The binding energies of multiple components of YXTMG to multiple targets of TLR4 pathway of COVID-19 pathway were low. Compared with the positive drug (prednisone), the panaxadiol in the compound showed better binding ability with zelactone A (fig 12a), indicating that YXTMG had good anti-inflammatory effect. The Pymol software was used for analysis and mapping of panaxadiol and IKBA, IKKB, and TLR4, and for analysis and mapping of zelactone $A$ and NFKB, and IRAK4. It was found that the compound was stably combined in the cavity of the target molecule, as shown in Fig. 12b.

\section{Effect of YXTMG on Expression of Target Molecular Proteins}

Western blot was used to detect the relative expression levels of TLR4 and NFKB in the COVID-19 pathway of the control group, the model group, the prednisone group, the quercetin group and the YXTMG group. It was found that the expression levels of TLR4 and NFKB in the model group were significantly higher than those in the control group $(p<0.05)$. Compared with the model group, the protein expression level of the prednisone group was decreased $(p<0.05)$. The expression of TLR4 was significantly reduced by $50 \mu \mathrm{M}$ quercetin $(p<0.01)$. The expressions of TLR4 and NFKB in the YXTMG group were decreased to different degrees, and the decreases were noted mostly in the three dosing groups (fig.13)

\section{Effect of YXTMG on Levels of Cytokines of AECQ Induced by Oxidative Injury}

To determine whether the drugs improved the activity of cells damaged by oxidation through immune pathway, TNF-a, IL- 6 and IL-10 of cells in each group were tested. The results showed that while compared with the control group, the levels of TNF- $a$ and IL- 6 in the model group were increased, while the level of IL-10 was decreased $(p<0.05)$. The levels of TNF- $a$ and IL- 6 in each administration group were decreased to different degrees, and the level of IL-10 was increased to different degrees in comparison with the model group (fig.14).

\section{Discussions}


Clinical studies in patients with COVID-19 (Chaolin Huang et al.,2020) have shown that cytokine storms may be a potential cause of aggravation of the diseased state in critically ill patients, which may be caused by innate immune dysfunction that causes hypoxia in patients with respiratory dysfunction.YXTMG has been proved to be effective in the treatment of angina pectoris. Recent study ( $\mathrm{Li}$ Nan,et al.,2021) has revealed that YXTMG can regulate PI3K-Akt pathway against myocardial injury in rats, so it has the possibility of preventing the deterioration of the disease by improving the immune function of patients with COVID-19. In COVID-19 patients, cytokine elevated level cause the release of oxygen free radicals, which damage cell function and further lead to the activation of inflammatory pathways (Erlich Jonathan et al.,2020). Cell model for oxidative stress was induced by using Hydrogen peroxide, as it acts as oxygen free radical donor, which ultimately stimulates oxidative stress process in vivo.

Through the analysis of differential miRNA expression in severe COVID-19 patients, we found that in terms of number, miRNA upregulation is dominant, but the miRNA that shows enormous amount of variations is basically down-regulated, while the patients with severe covid-19 infection showed higher expression level of mRNA, when compared with milder patients, which may be mediated by excessive upregulation of miRNA. As shown in supplement Fig. 3a, IL10, CD4, FGF2, IL1ß, CD274, MMP1, and CXCL10 are the primary regulatory genes in patients, most of which are important molecules for regulating inflammatory pathways. Secondly, through GO analysis, it was found that biological processes such as T cell-mediated immunity, acute inflammatory response, $\mathrm{B}$ cell activation, and neutrophil degranulation in severe patients have been changed, which is consistent with the pathological characteristics such as oxidative stress and immune response found in patients in one study (Singh HariOm et al., 2021). Pathway analysis of differential genes revealed that these functional changes were possibly related to cell adhesion molecule, cytokine-cytokine receptor interaction, NF-KB and TNF pathways in environmental information processing, and also closely related to Th1, Th2 and Th17 cell differentiation, natural killer cell-mediated cytotoxicity, complement system and coagulation cascade pathways in the tissue system. These results suggested that the above signaling pathways could serve as intervention pathways to change oxidative stress and inflammatory state in patients.

In this study, the network pharmacology was used to predict the molecular mechanism of YXTMG in the treatment of patients with COVID-19. Eighty-three constituents of YXTMG, including quercetin, kaempferol and zelactone A, were collected and showed interaction with 375 targets in COVID-19, with STAT family, interleukin family and NF-KB family as the important targets, and flavonoids, terpenes, steroids and alkaloids as the main active entities of YXTMG. Gene ontology and pathway enrichment analysis of the intersecting targets revealed that the prescription of active compounds might have role in biological processes such as cytokine production, $T$ cell activation, leukocyte chemotaxis, and active oxygen metabolism by regulating signaling pathways such as Th17 cell differentiation, HIF1, PI3K-Akt, and Tolllike receptor. At present, PLAU and SERPINE1 are considered to be new targets for the treatment of myocardial infarction (Lippi Giuseppe,et al.,2021;Hisada Yohei,et al.,2021). Meanwhile, up-regulation of miR-181a-5p has a role in protecting myocardial tissue (CAl Lu-ming,et al.,2021). High performance liquid chromatography showed that quercetin was present in YXTMG and its content was high. Intersection 
analysis of differential genes between YXTMG and miRNA-mediated mRNA revealed that, the quercetin and stigmasterol causes the down-regulation of miR-181a-5p, which might induces the interference with the up-regulation of PLAU and SERPINE1 by being playing thrombolytic role by the compounds, which was of great significance for delaying the worsening of COVID-19. Analysis of the effect of the combination of gene phenotype of severe patients and YXTMG revealed that the drug might affect the biological processes of acute inflammation, cytokine secretion, and $T$ cell immunity of the patient by regulating Th1, Th2, and Th17 cell differentiation, NF-KB signaling pathways, and at the same time, the drug might improve the hypoxia and response to oxygen level by interfering in the miRNA-mediated downregulation of PI3K-Akt pathway, thereby showing the anti-COVID-19 effect. In short, YXTMG may regulate the processes of hypoxia and oxidative stress, intravascular coagulation and acute inflammation in patients with COVID-19.

Under physiological conditions, type II alveolar epithelial cells can proliferate and differentiate into type I alveolar epithelial cells and secrete phospholipid-rich pulmonary surfactant, which reduces alveolar surface tension to maintain the structural integrity of alveolar wall (Song Lin,et al.,2021). When lung injury factors persist, the proliferation and differentiation of type II alveolar epithelial cells are significantly reduced, resulting in respiratory dysfunction. This experimental study simulated the physiological process of cytokines producing excessive oxygen free radicals and damaging type II alveolar epithelial cells. The blood inlet component of YXTMG was used to administer hydrogen peroxide to induce AECQ injury. The results showed that YXTMG could significantly improve cell activity, indicating that YXTMG had certain value for the development of anti-inflammatory drugs. The evaluation of the drug's oxygen free radical scavenging capacity showed that both YXTMG and quercetin had good antioxidant activity. The detection of the expression levels of TLR4, NFKB, IL-6, TNF-a, and IL-10 revealed that YXTMG significantly inhibited the activation of Toll receptor in COVID-19 pathway. The relevant targets are shown in Fig. 15. Molecular docking was used to verify the action potential of the important components against the targets, and it was found that many components of YXTMG, such as panaxadiol, zelactone $A$, and kaempferol, had potential anti-inflammatory biological activities. All these results indicated that YXTMG had good improving effect on cell damage caused by oxidative stress, and it has the potential to be used for the treatment of COVID-19.

\section{Conclusion}

In summary, Our study has further confirmed that severe COVID-19 is closely related to oxidative stress, hypoxia, immune disorders and thrombosis, which may provide a reference for target screening and drug development. Based on network pharmacology and serum pharmacology, we screened out Chinese patent medicine, which has regulatory potential in the above etiology, and can be used as a candidate drug for further development and application in critically ill patients.

\section{Declarations}

\section{Author contributions}


CLF, ZYZ contributed to study design, data interpretation, and manuscript preparation. WY, LMJ contributed to data acquisition.

\section{Funding}

This work was supported by the National Natural Science Foundation of China (81960661).

\section{Acknowledgments}

The authors would like to acknowledge Xinjiang Medical University for Biological information platform, experimental platform, and database for finish this manuscript.

\section{Availability of data and materials}

The datasets for this study can be found in GEO databases [https://www.ncbi.nlm.nih.gov/geo/].

\section{Ethics approval and consent to participate}

Study design experiments approved ethically from Xinjiang Medical University.

\section{Consent for publication}

Not applicable

\section{Competing interests}

The authors declare that the research was conducted in the absence of any commercial or financial relationships that could be construed as a potential or actual conflict of interest.

\section{References}

1. CAI Lu-ming, Hou Mei-ling, Chao Ya-wei, et al. Effects of lentivirus-mediated miR-181a-5p overexpression on cardiac remodeling in rats with chronic heart failure [J]. Prevention and treatment of cardiovascular and cerebrovascular diseases,2021,21(04):338-341+349.

2. CAI Mei-yun, Zhuang Wen-xin, Lu E, et al. Kaempferol reduces PC12 cell inflammation induced by 6hydroxydopamine (6-OHDA) by inhibiting p38 MAPK pathway [J]. Journal of Cell and Molecular Immunology, 2020,36(07):583-589.

3. Chaolin Huang et al. Clinical features of patients infected with 2019 novel coronavirus in Wuhan, China[J]. The Lancet, 2020, 0(prepublish) : 497-506.

4. Chen Panther, Liu Huiguo, Liu Wei, et al. 2019 Analysis of clinical characteristics of 29 cases in novel coronavirus [J]. Chinese Journal of Tuberculosis and Respiratory Medicine, 2020(03):203-208.

5. CHEN Yan. Study on the molecular mechanism of panaxadiol saponins in improving the renal function of LPS-induced acute kidney injury mice [D]. Jilin University, 2014. 
6. Erlich Jonathan et al. Targeting evolutionary conserved oxidative stress and immunometabolic pathways for the treatment of respiratory infectious diseases.[J]. Antioxidants \& redox signaling, 2020, 32(13) : 993-1013.

7. Hisada Yohei,Garratt Kenison B.,Maqsood Anaum,et al. Plasminogen activator inhibitor 1 and venous thrombosis in pancreatic cancer[J]. Blood Advances,2021,5(2):1-9.

8. Jiang Xin. Yixin Tongmai Granule in the treatment of stable angina pectoris qi deficiency and blood stasis syndrome clinical analysis of 49 cases. Chinese community physicians. 2012, 28(40):16.

9. Li Nan, Hong Linwei, Cong Jing, et al. Study of myocardial injury and stress level of Yixin Tongmai Granule on the basis of PI3K/AKT pathway in CHD rats [J]. Journal of translational medicine, 2021,10(04):211-216.

10. Lippi Giuseppe,Henry Brandon M,Favaloro Emmanuel J. Elevated soluble urokinase plasminogen activator receptor (suPAR) in COVID-19 patients.[J]. Clinical chemistry and laboratory medicine, 2021, 59(11) : e413-e415.

11. L N, WG W, G P, et al. Neutrophil/Lymphocyte ratio is associated with non-calcified plaque burden in patients with coronary artery disease. PloS one. 2014,9(9):1-9.

12. Ma Shan-shan,Xie Zhong-yang,Zhang Ling-jian,et al. Identification of a Potential miRNA-mRNA Regulatory Network Associated With the Prognosis of HBV-ACLF[J]. Frontiers in Molecular Biosciences,2021,8:10.

13. Ma Shaodan, Bai Jing, Cheng Yuanpeng, et al. An Experimental Study on the Anti-inflammatory Effect of Jiu Xie Ning Granules [J]. Shi Zhen Guo Yi Guo Yao, 2016,27(08):1841-1842.

14. Metcalf JV, Tang Dezhi, Shu Bing, et al. Exploration of the mechanism of Chinese medicine intervention on novel coronavirus based on literature [J]. journal of traditional chinese medicine, 2020,61(13):1110-1117.

15. R R, J G, B K, et al. A platelet-dependent serum factor that stimulates the proliferation of arterial smooth muscle cells in vitro. Proceedings of the National Academy of Sciences of the United States of America. 1974,71(4):1207-1210.

16. Singh HariOm et al. Immune mediating molecules and pathogenesis of COVID-19-associated neurological disease[J]. Microbial Pathogenesis, 2021, 158 : 105023-105023.

17. Song Lin,Peng Juan,Guo Xuejun. Exosomal IncRNA TCONS_00064356 derived from injured alveolar epithelial type II cells affects the biological characteristics of mesenchymal stem cells[J]. Life Sciences,2021,278:1.

18. Zhu Yanqi et al. Reduced levels of circulating CD4+CD25+CD127lo regulatory $T$ cell in patients with acute myocardial infarct predict a high risk of reccurrence of angina pectoris during postinfarction period[J]. CIRCULATION, 2012, 125(19) : E725-E726.

19. Zhan Jin-shun, Huo Jun-hong, Zhan Kang, et al. Effects of quercetin on antioxidant and antiinflammatory activities of goat rumen epithelial cells stimulated by lipopolysaccharide [J]. grassland science, 2021,38(07):1393-1401. 


\section{Tables}

Table 1 Main active compounds of YXTMG 


\begin{tabular}{|c|c|c|c|}
\hline Traditional Chinese Medicine & Compound & OB (\%) & $\mathrm{DL}$ \\
\hline \multirow[t]{5}{*}{ Glehniae Radix } & quercetin & 46.43 & 0.28 \\
\hline & isoimperatorin & 45.46 & 0.23 \\
\hline & Stigmasterol & 43.83 & 0.76 \\
\hline & Bergaptin & 41.73 & 0.42 \\
\hline & beta-sitosterol & 36.91 & 0.75 \\
\hline \multirow[t]{5}{*}{ Chuanxiong Rhizoma } & FA & 68.96 & 0.71 \\
\hline & Perlolyrine & 65.95 & 0.27 \\
\hline & senkyunone & 47.66 & 0.24 \\
\hline & wallichilide & 42.31 & 0.71 \\
\hline & Mandenol & 42 & 0.19 \\
\hline \multirow[t]{5}{*}{ Radix Salviae } & digallate & 61.85 & 0.26 \\
\hline & $\begin{array}{l}\text { (E)-3-[2-(3,4-dihydroxyphenyl)-7-hydroxy- } \\
\text { benzofuran-4-yl]acrylic acid }\end{array}$ & 48.24 & 0.31 \\
\hline & isoimperatorin & 45.46 & 0.23 \\
\hline & 3a-hydroxytanshinone『a & 44.93 & 0.44 \\
\hline & Poriferasterol & 43.83 & 0.76 \\
\hline \multirow[t]{5}{*}{ Hedysarum Multijugum Maxim } & isoflavanone & 109.99 & 0.3 \\
\hline & 7-0-methylisomucronulatol & 74.69 & 0.3 \\
\hline & formononetin & 69.67 & 0.21 \\
\hline & FA & 68.96 & 0.71 \\
\hline & $\begin{array}{l}\text { (3R)-3-(2-hydroxy-3,4-dimethoxyphenyl)chroman- } \\
\text { 7-ol }\end{array}$ & 67.67 & 0.26 \\
\hline \multirow[t]{5}{*}{ Panax Ginseng C. A. Mey } & Celabenzine & 101.88 & 0.49 \\
\hline & Aposiopolamine & 66.65 & 0.22 \\
\hline & Frutinone A & 65.9 & 0.34 \\
\hline & Inermin & 65.83 & 0.54 \\
\hline & Girinimbin & 61.22 & 0.31 \\
\hline \multirow[t]{2}{*}{ Figwort Root } & harpagoside_qt & 122.87 & 0.32 \\
\hline & paeoniflorin_qt & 68.18 & 0.4 \\
\hline
\end{tabular}




\begin{tabular}{llll} 
& 14-deoxy-12(R)-sulfoandrographolide & 62.57 & 0.42 \\
\hline & scropolioside A_qt & 38.63 & 0.77 \\
\hline beta-sitosterol & 36.91 & 0.75 \\
\hline \multirow{3}{*}{ Curcumae Radix } & Zedoalactone A & 111.43 & 0.19 \\
\hline & zedoalactone E & 85.16 & 0.19 \\
\hline Oxycurcumenol & 67.06 & 0.18 \\
\hline (E)-1,7-Diphenyl-3-hydroxy-1-hepten-5-one & 64.66 & 0.18 \\
\hline Radix Glycyrrhizae Preparata & (4aR,5R,8R,8aR)-5,8-dihydroxy-3,5,8a-trimethyl- & 59.52 & 0.2 \\
\hline & 6,7,8,9-tetrahydro-4aH-benzo[f]benzofuran-4-one & & \\
\hline 18beta-Glycyrrhetinic Acid & 22.5 & 0.74 \\
\hline & Glycyrrhetinic Acid & 16.21 & 0.76 \\
\hline & Glycyrrhizin & 19.62 & 0.11 \\
\hline & 18alpha-Glycyrrhetinic Acid & 17.41 & 0.74
\end{tabular}

Table 2 Topology analysis of interaction network between YXTMG and COVID-19

\begin{tabular}{lll} 
Compound & Degree & Source \\
\hline Quercetin & 236 & Hedysarum Multijugum Maxim \\
\hline Kaempferol & 88 & Panax Ginseng C. A. Mey \\
\hline Beta-sitosterol & 84 & Curcumae Radix \\
\hline Gomisin B & 66 & Panax Ginseng C. A. Mey \\
\hline (3R)-3-(2-hydroxy-3,4-dimethoxyphenyl)chroman-7-ol & 63 & Hedysarum Multijugum Maxim \\
\hline Isoflavanone & 59 & Hedysarum Multijugum Maxim \\
\hline (E)-5-Hydroxy-7-(4-hydroxyphenyl)-1-phenyl-1-heptene & 56 & Curcumae Radix \\
\hline (E)-1,7-Diphenyl-3-hydroxy-1-hepten-5-one & 56 & Curcumae Radix \\
\hline Celabenzine & 56 & Panax Ginseng C. A. Mey \\
\hline Oxycurcumenol & 55 & Curcumae Radix
\end{tabular}

Table 3 Quantitative analysis of quercetin 


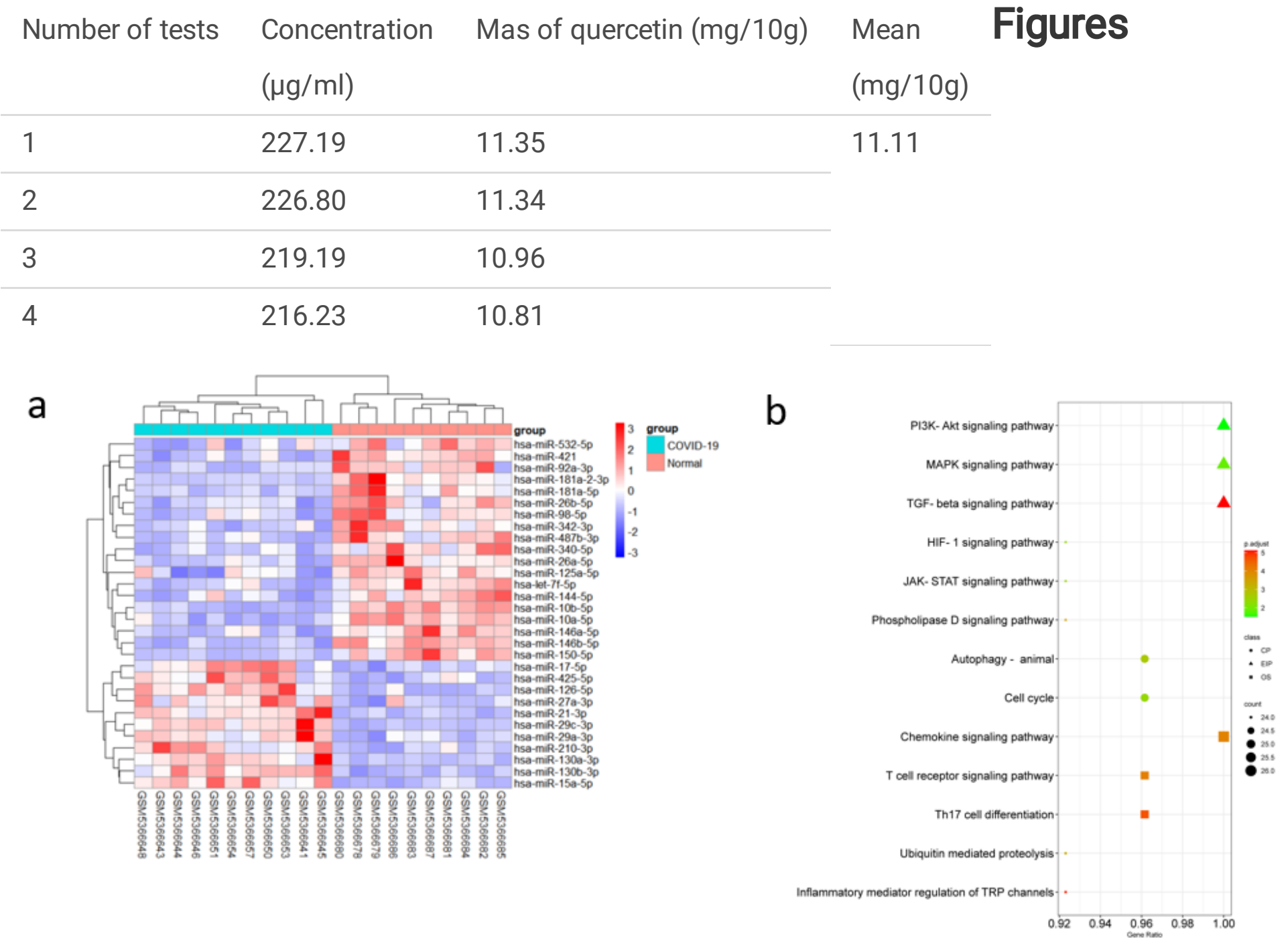

\section{Figure 1}

heat map of microRNA expression(a) in critical COVID-19 patients and its enrichment pathway(b). 


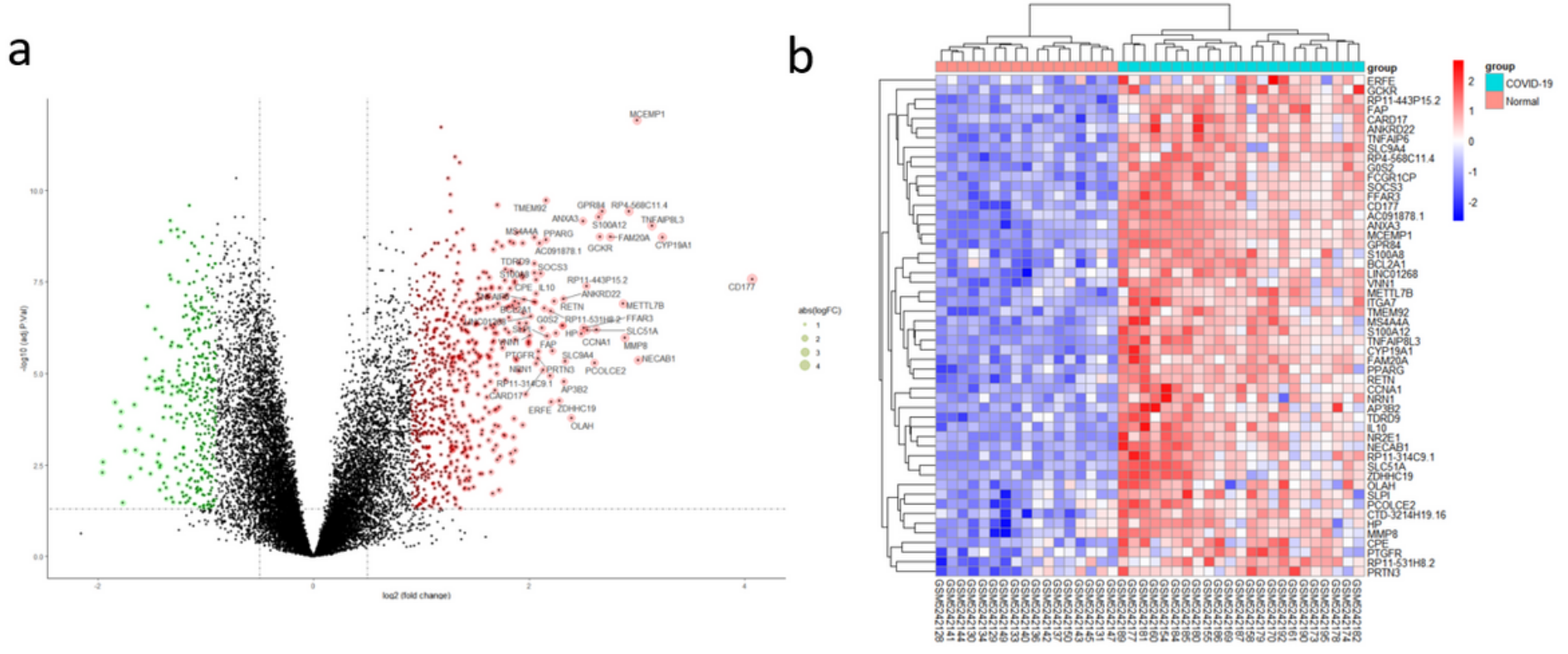

Figure 2

Volcano(a) and heat diagram(b) of mRNA expression in severe COVID-19 patients 

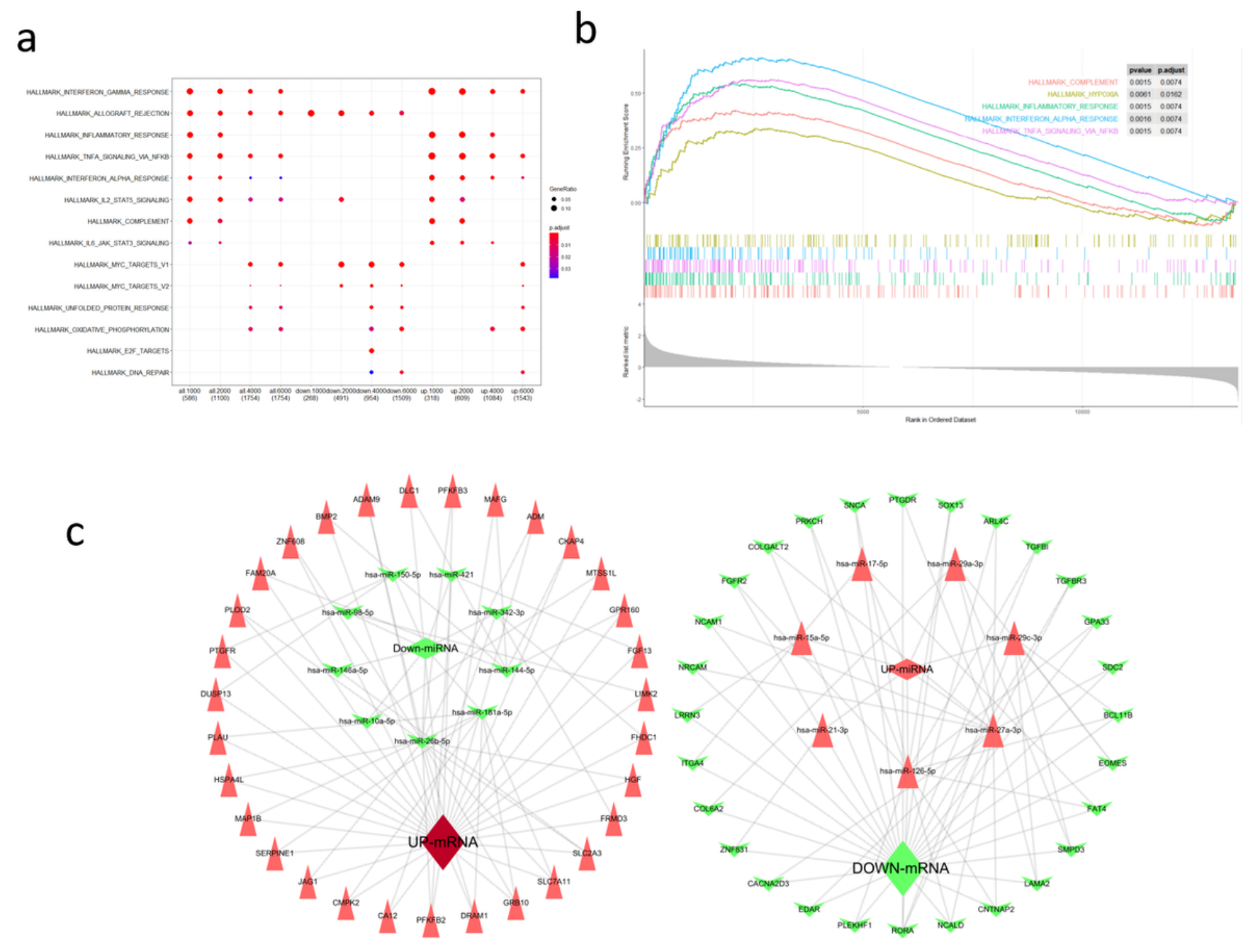

\section{Figure 3}

Bubble diagram (a), enrichment fraction curve (b) and miRNA regulatory mRNA network (c) of gene set enrichment analysis 
a

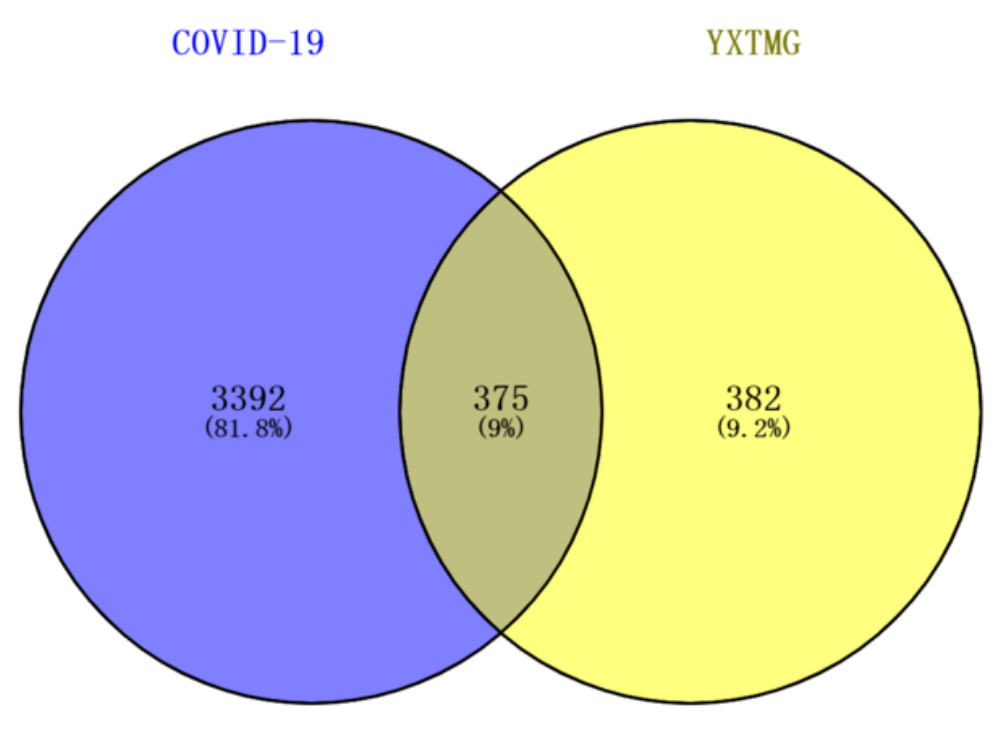

\section{Figure 4}

Analysis of targets of YXTMG in the treatment of COVID-19 b

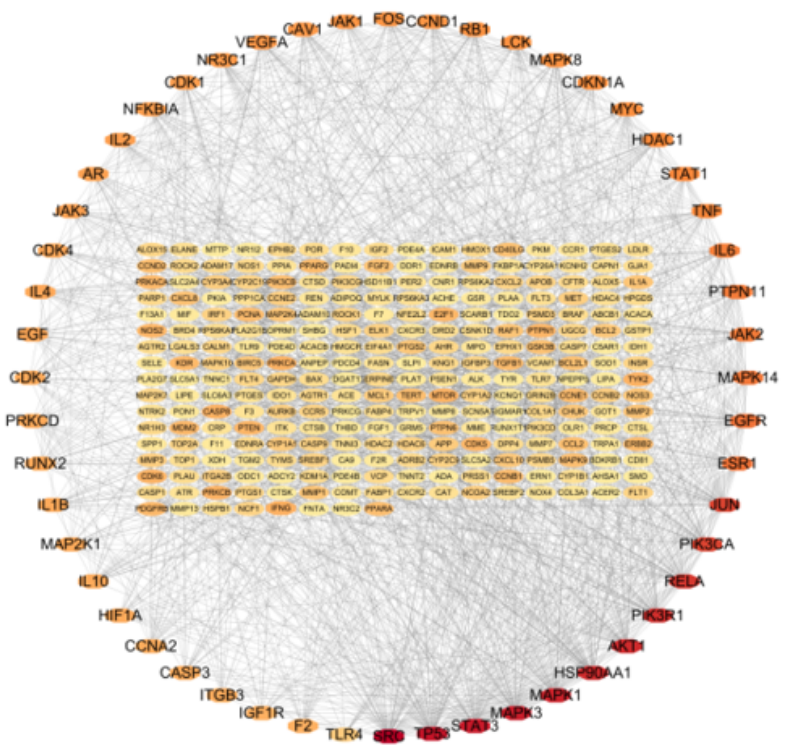


a
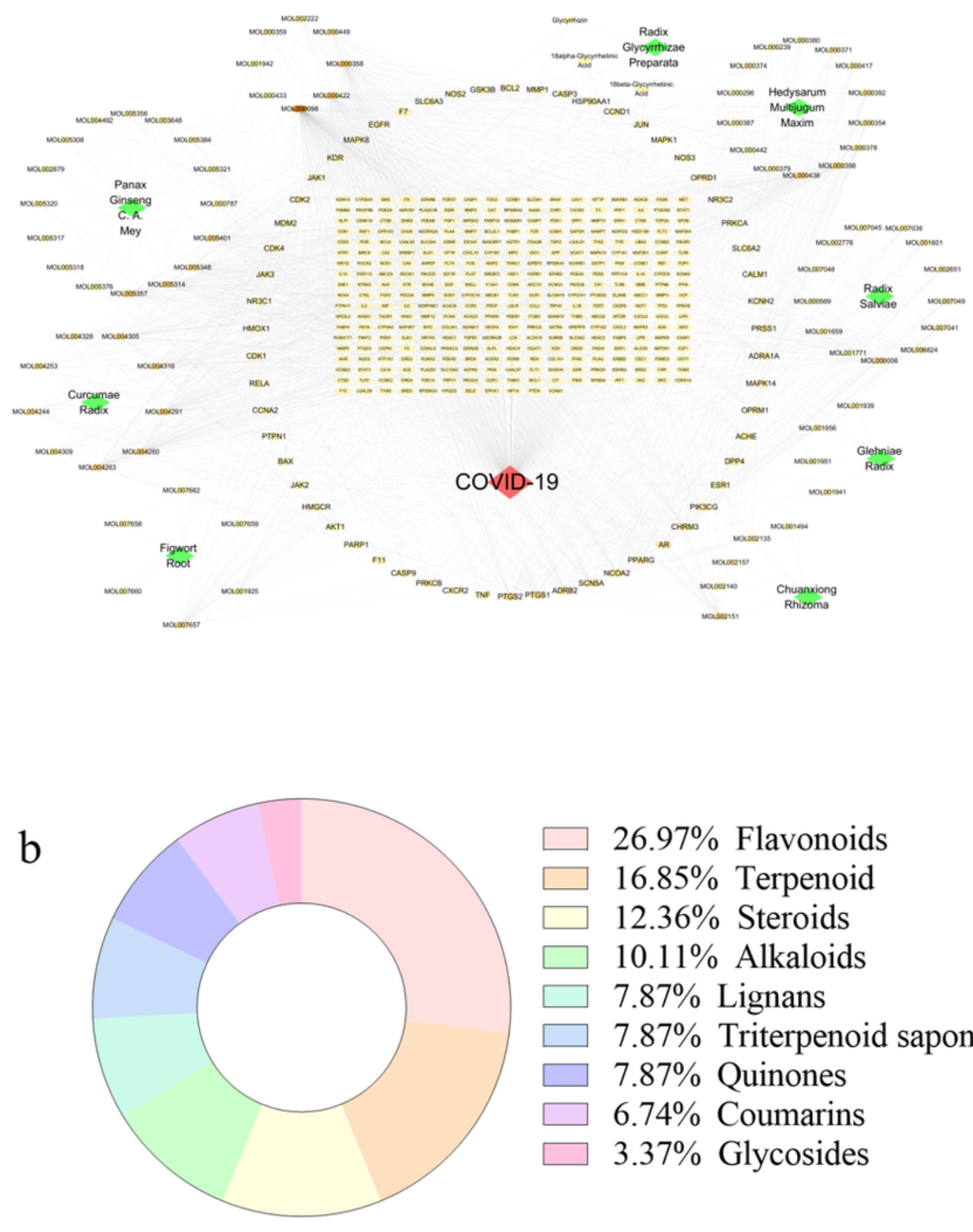

26.97\% Flavonoids

$16.85 \%$ Terpenoid

$12.36 \%$ Steroids

$10.11 \%$ Alkaloids

$7.87 \%$ Lignans

$7.87 \%$ Triterpenoid saponin

$7.87 \%$ Quinones

$6.74 \%$ Coumarins

$3.37 \%$ Glycosides

Figure 5

Drug-Disease Network Diagram(a) and Component Analysis Summary(b) 

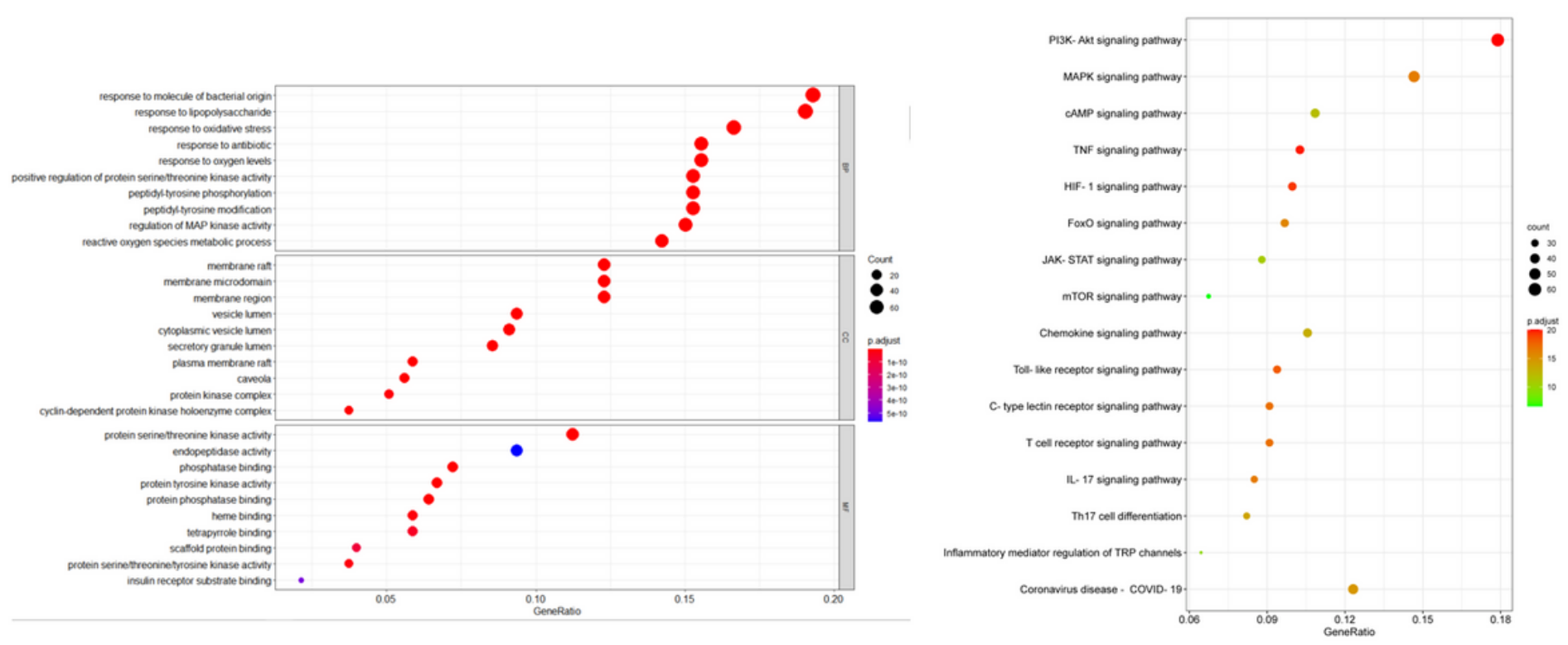

Figure 6

Genetic ontology and pathway analysis of COVID-19 treated with YXTMG
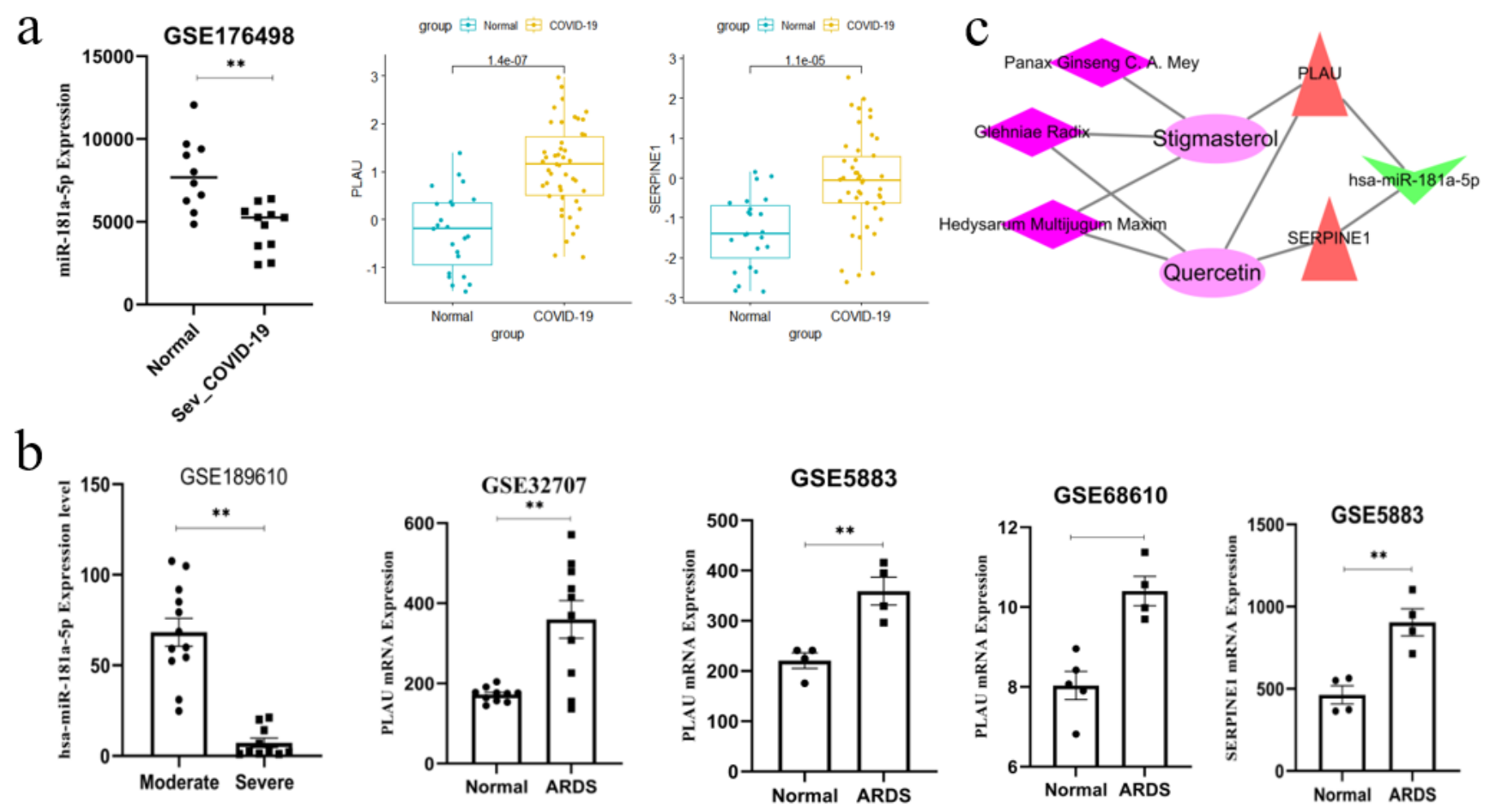

\section{Figure 7}

Expression Profiling(a) and Dataset Validation(b) of miR-181a-5p, PLAU, and SERPINE1, and Analysis of Drug Mechanism(c) 

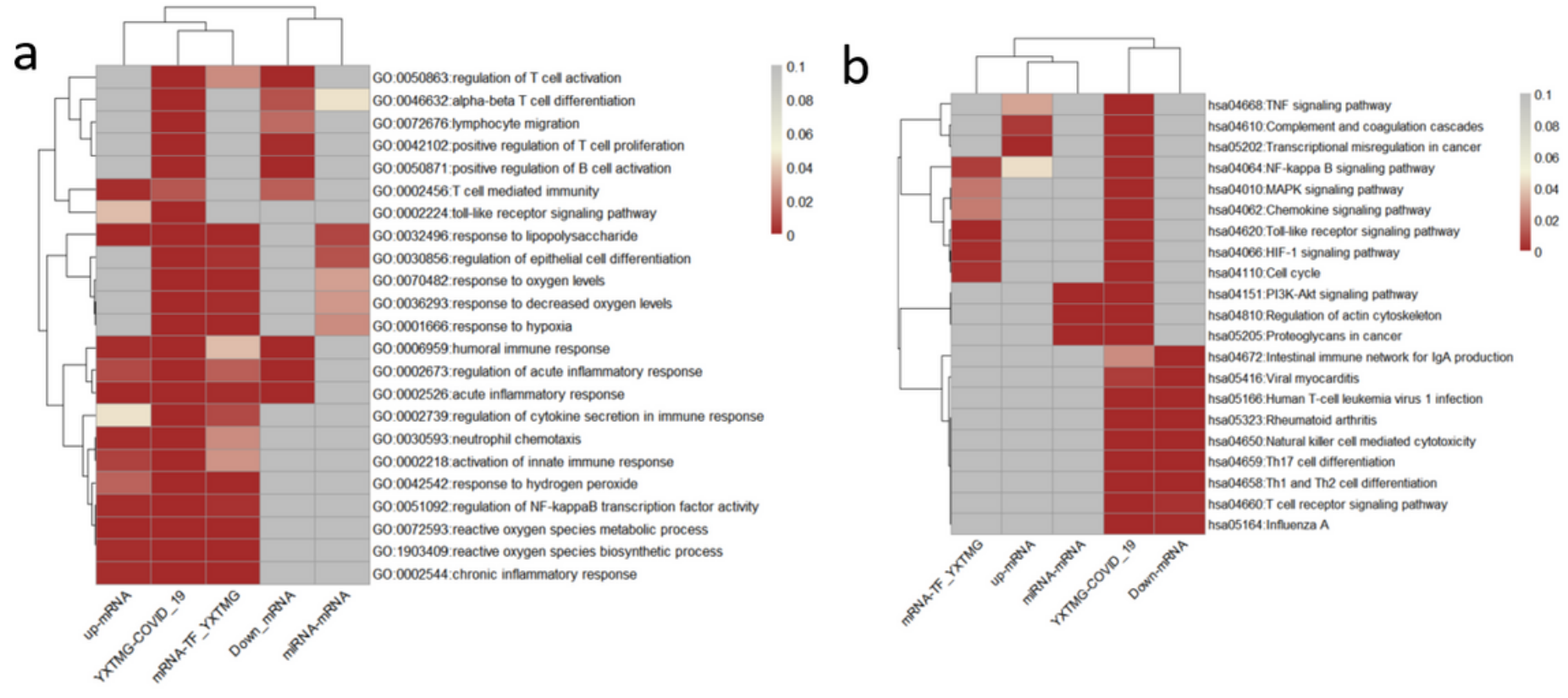

\section{Figure 8}

Biological process(a) and pathway(b) combination analysis of differential miRNA-mRNA, up-regulation and down-regulation mRNA and YXTMG in the treatment of COVID-19 
$\mathrm{a}$

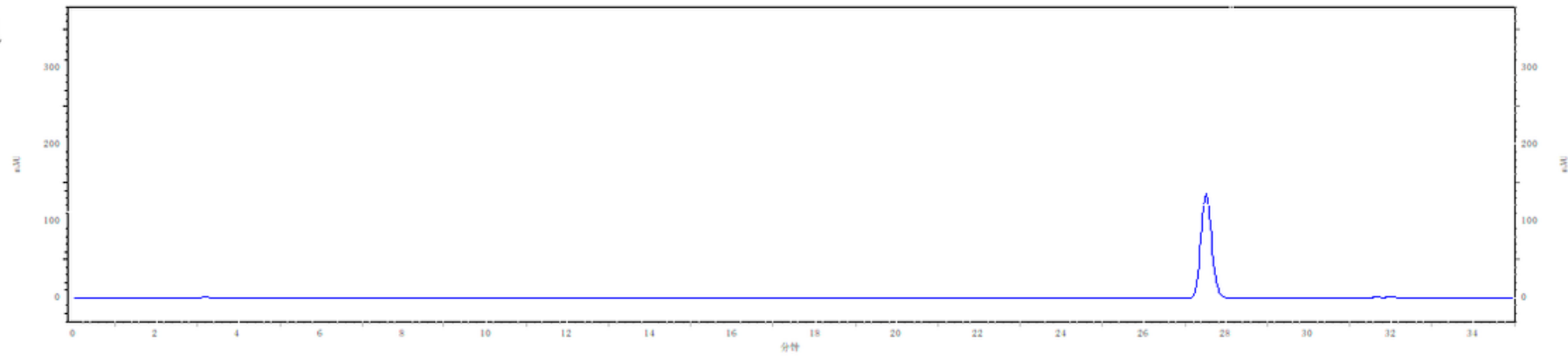

b

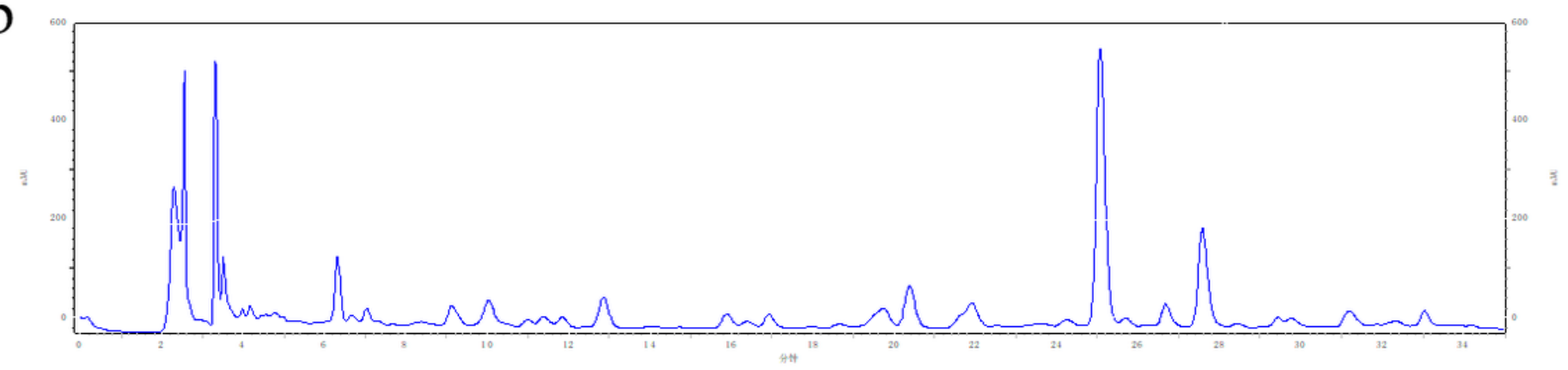

C

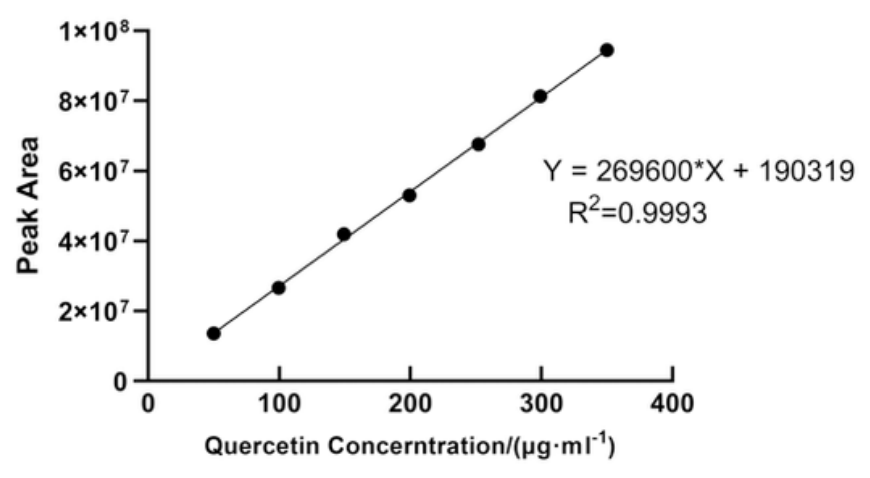

Figure 9

Chromatogram of quercetin(a) and YXTMG(b) , and working curve(c) of quercetin 

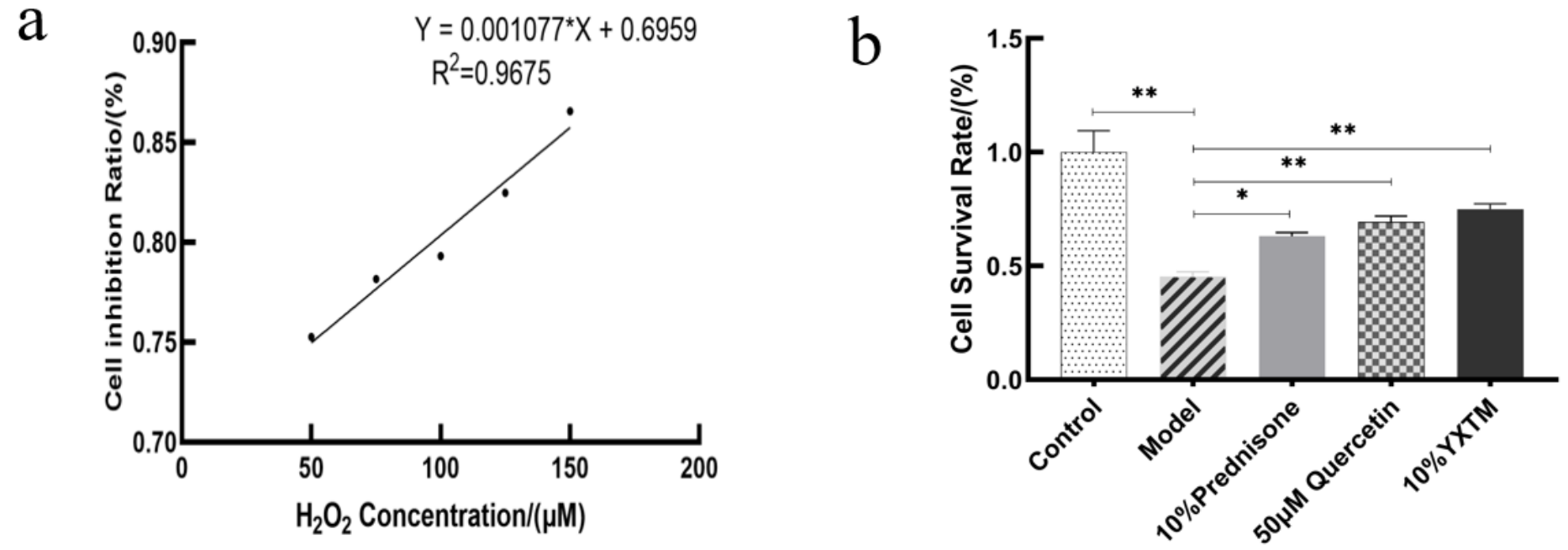

Figure 10

Establishment of oxidative stress model induced by hydrogen peroxide and the effect of drugs on cell viability after oxidative damage activity

a

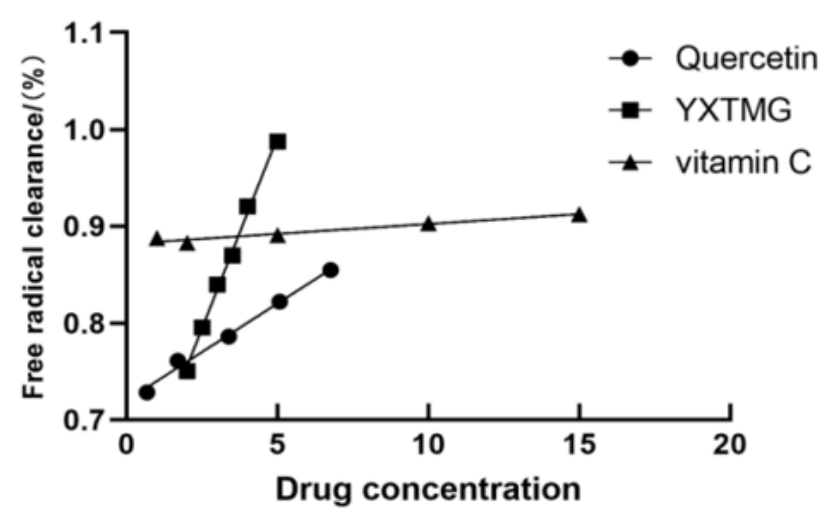

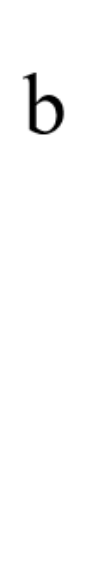

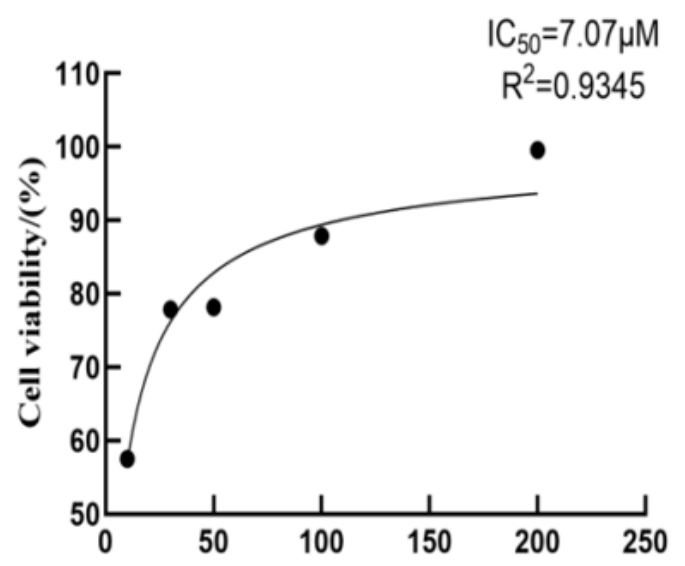

\section{Figure 11}

Evaluation of free radical clearance(a) and determination of Quercetin IC50(b) 


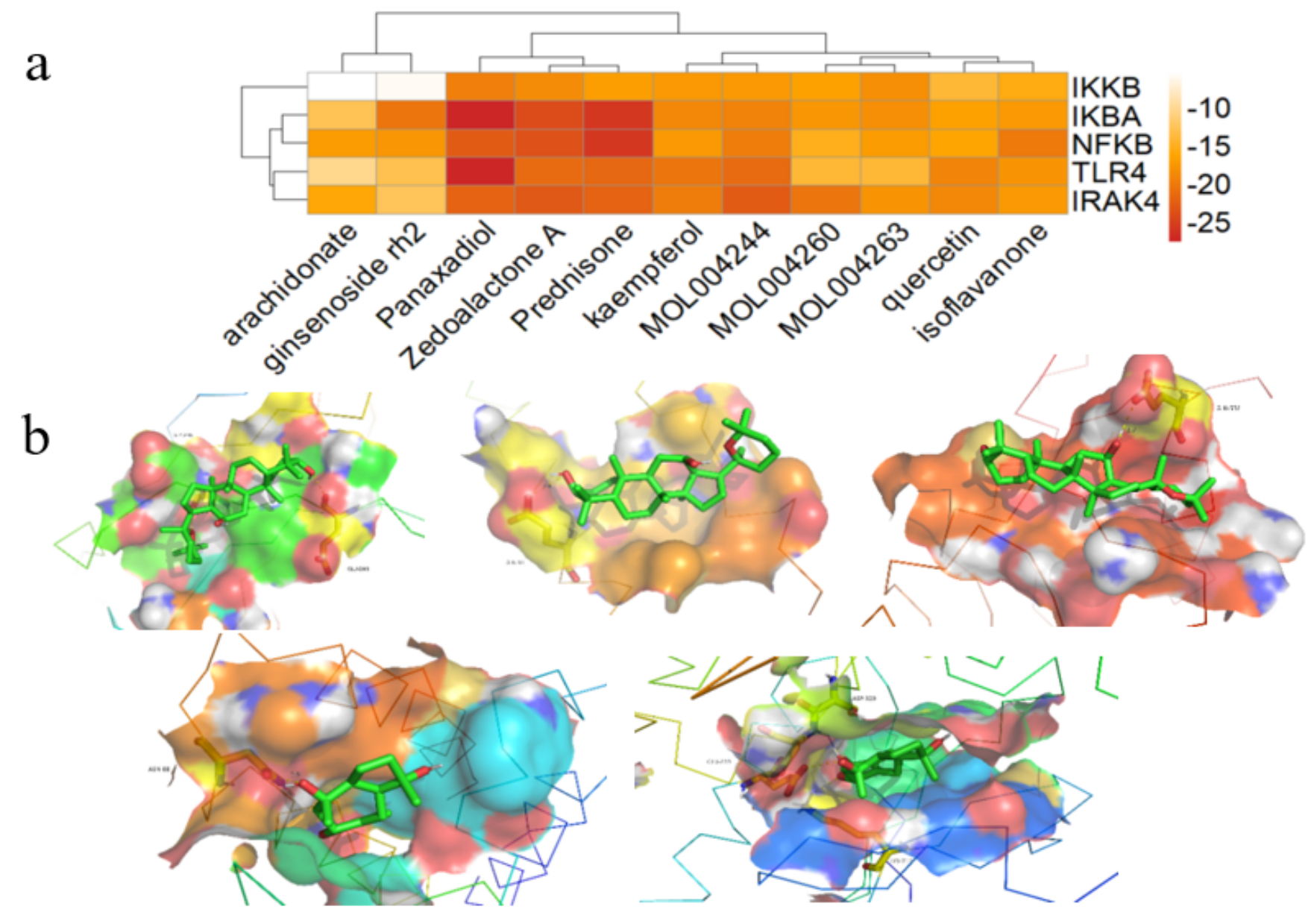

Figure 12

heat map of molecular docking results and optimal binding site map 
TLR4

$\mathrm{NF} \kappa \mathrm{B}$

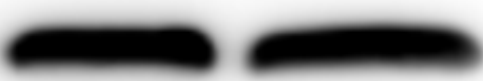

a

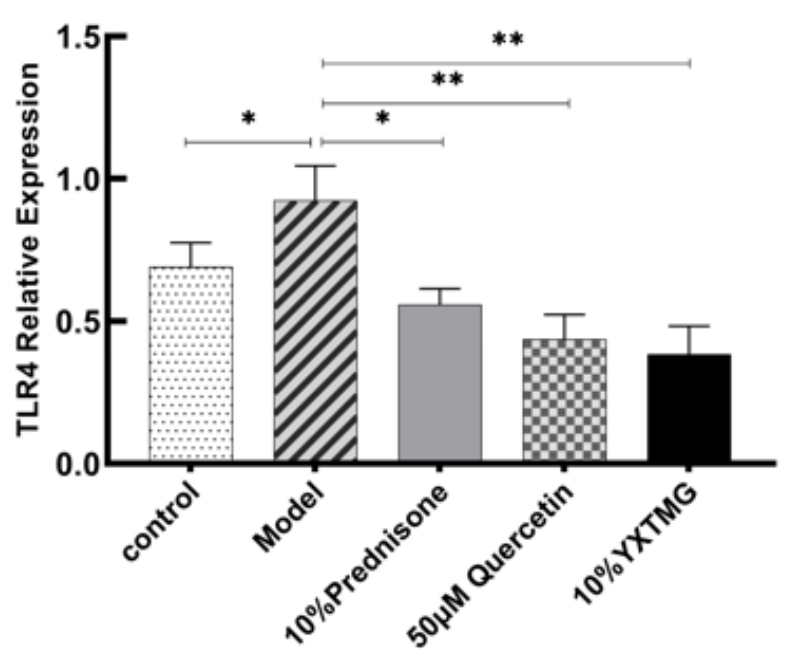

b

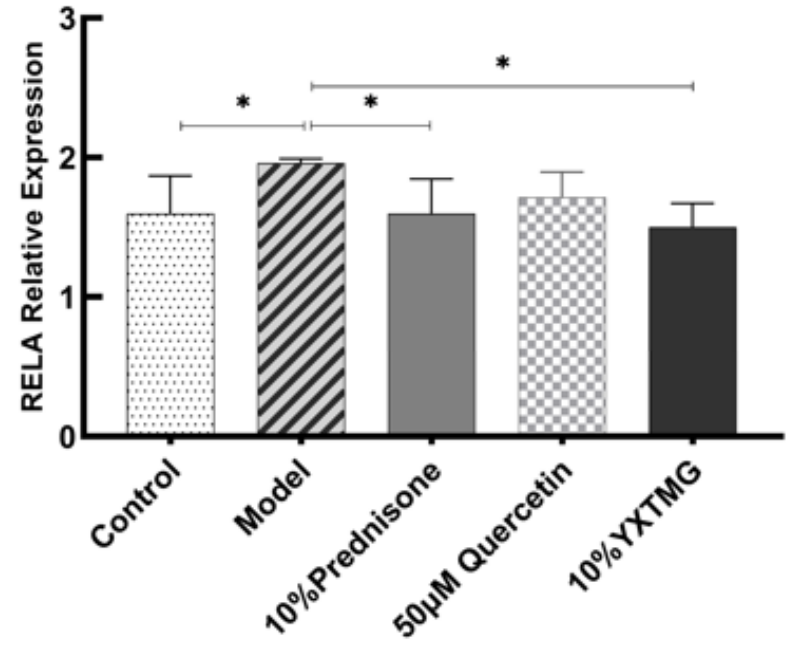

Figure 13

Effects of different dosing groups on the expression of TLR4(a) and NFKB(b) in oxidative injury cell
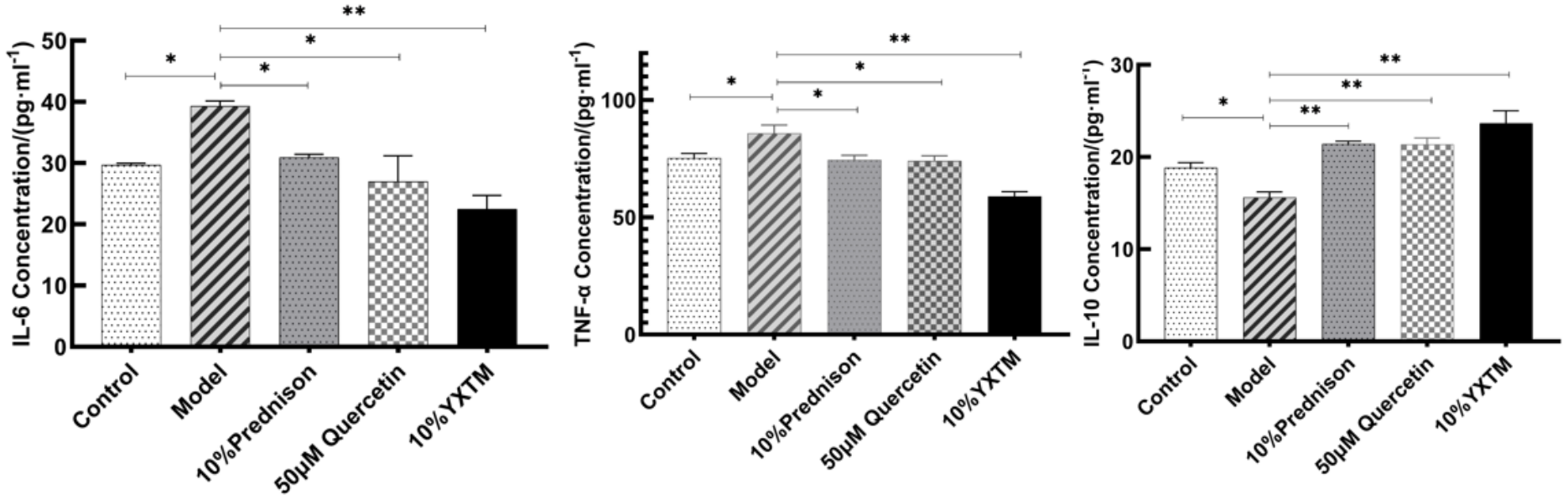

Figure 14

Effect of YXTMG on cytokines of AECQ induced by oxidative Injury $\star: p<0.05,{ }^{* *}: p<0.01$. 


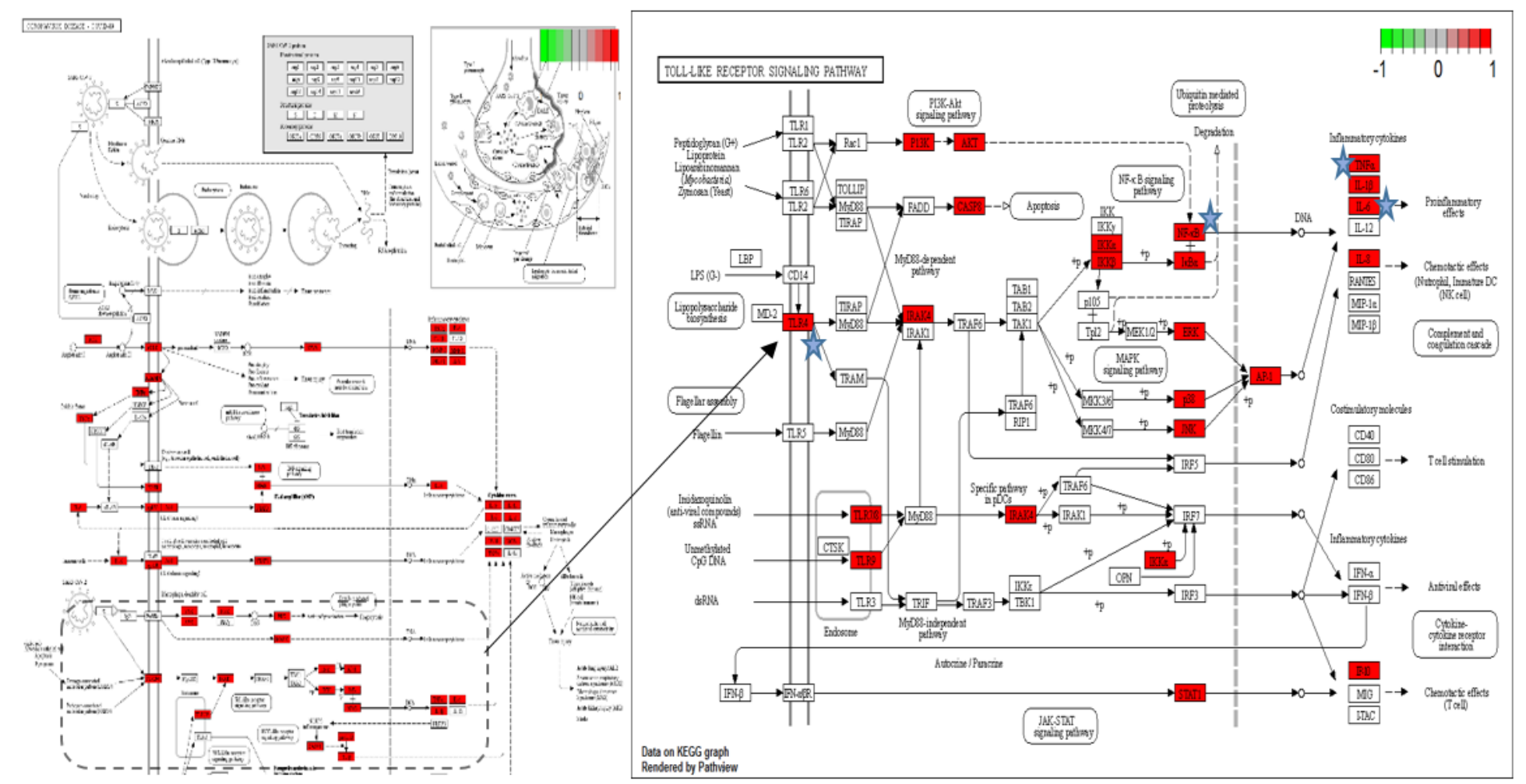

Figure 15

COVID-19 and Toll-like receptor signaling pathway diagram

\section{Supplementary Files}

This is a list of supplementary files associated with this preprint. Click to download.

- Supplementmaterial.docx 